\title{
In Vitro Study of Adipose-Derived Mesenchymal Stem Cells Transduced with Lentiviral Vector Carrying the Brain-Derived Neurotrophic Factor Gene
}

\author{
De Nan ${ }^{1}$, Xiang Dou ${ }^{1}$, Yankai Qi ${ }^{1}$, Wenjun Zhang ${ }^{1}$, Gaohong $\mathrm{He}^{1,2}$, Xiujuan Zhang ${ }^{1}$ \\ ${ }^{1}$ State Key Laboratory of Fine Chemicals, School of Chemical Engineering, Panjin Campus, Dalian University of Technology, Panjin, China \\ ${ }^{2} R E D$ Center of Membrane Science and Technology, School of Chemical Engineering, Dalian University of Technology, Dalian, China
}

Brain-derived neurotrophic factor (BDNF) exerts its survival-promoting effects on photoreceptors and retinal ganglion cells, however, delivery systems with little-to-no side effect are needed to sustain its controlled release and long-term efficacy. Our previous studies demonstrated that adipose-derived stem cells (ADSCs) are ideal delivery systems for gene therapy; moreover, ADSCs present unique properties like migration to damaged tissue sites, immunomodulation and anti-inflammation. Herein, we propose to employ ADSCs as the BDNF gene delivery vehicle. Different Analyses like flow cytometry, differentiation and cell proliferation assays etc demonstrated that BDNF were successfully transduced into ADSCs and the stemness of ADSCs was maintained even with the transduction. Real Time PCR and Western blot were used to measure mRNA and protein expressions of the BDNF-transduced ADSCs. The results demonstrated that the BDNF expression level of the lentiviral-BDNF transduced ADSCs is significantly increased and, moreover, enhanced the expression of other neurotrophic and downstream signaling factors. The data suggest that ADSCs are a reliable delivery vehicle for BDNF and could be used for the treatment of various diseases.

Keywords: Brain-derived neurotrophic factor, Adipose-derived mesenchymal stem cells, Lentiviral transduction, Drug delivery, Neural protection

\section{Introduction}

Brain-derived neurotrophic factor (BDNF), a member of neurotrophic factor family, was first isolated from pig

Received: March 7, 2020, Revised: July 13, 2020,

Accepted: July 13, 2020, Published online: August 31, 2020

Correspondence to Xiujuan Zhang

State Key Laboratory of Fine Chemicals, School of Chemical Engineering, Panjin Campus, Dalian University of Technology, Panjin 124221, China

Tel: +86-427-2631960, Fax: +86-427-2631960

E-mail: xiujuanzhang@dlut.edu.cn

(a) This is an open-access article distributed under the terms of the Creative Commons Attribution Non-Commercial License (http://creativecommons.org/ licenses/by-nc/4.0/), which permits unrestricted non-commercial use, distribution, and reproduction in any medium, provided the original work is properly cited.

Copyright (c) 2020 by the Korean Society for Stem Cell Research brain extract by German neurobiologist BARDE in 1982. BDNF has a larger distribution of its mRNA-expressing cells and a wider distribution of receptors in comparison to other neurotrophic factors $(1,2)$. Researches have confirmed that BDNF has the ability to inhibit the progression of neuronal degenerations, plays an important role in neuronal survival and maintenance of the biological actions by activation of its intracellular pathways and relative receptors, especially its cognitive receptorTrkB (3). Although the biological effect of BDNF on neuronal survival and its physiological mechanism has been researched systematically, broader applications of BDNF requires exogenous $\mathrm{BDNF}$ injection because the endogenous BDNF expression in diseased models is much lower than that of the normal controls (4). However, exogenous BDNF injected with systemic administration can't penetrate the blood-retina or blood-brain barrier due to its size 
(5) and direct local injection, although effective, cause serious side effects. For example, although intravitreal injection of BDNF is effective for the treatment of retinitis pigmentosa (1), repeated injection is needed due to its short half-life in vivo, which easily causes risks of endophthalmitis and retinal detachment (6). Therefore, finding a reliable exogenous BDNF delivery system is the key for wider clinical applications (7).

Adipose-derived mesenchymal stem cells (ADSCs) are a population of mesenchymal stem cells (MSCs) isolated from adipose tissue. Like any other MSCs, ADSCs are featured with adherence to plastics, expressing specific cell surface markers and having multipotent differentiation potential (8). Moreover, ADSCs are attracting more and more attention for its unique advantages. For example, the amount of ADSCs obtained from adipose tissue is 100 to 1,000 times that of bone marrow stem cells in the same volume of tissue $(9,10)$. Meanwhile, the acquisition of adipose tissue is much simpler with less invasive and painful operation, such as abdominal fat aspiration. Therefore, ADSCs can be easily obtained in large quantities, cultured and expanded in vitro. Researches have shown that ADSCs have the potential to migrate to injured tissue sites, and provide therapeutic repair of the damaged tissues. Implantation of ADSCs could reduce immune rejections due to its low immunogenicity $(11,12)$. What's more, it has been reported that ADSCs can successfully pass the blood-brain or blood-retina barrier. Therefore, ADSCs are attractive candidates in gene delivery systems (13). In addition to act as a gene delivery vehicle, the therapeutic effects of ADSCs themselves by replacing damaged cells through cellular differentiation, cellular factor secretion, regulation of inflammatory cytokines, and creation of favorable environment for regeneration will be synergistic to the gene therapy efforts.

Herein, in consideration of the protection effects of BDNF on nerve cells and the advantages of ADSCs as a gene delivery carrier, murine ADSCs were isolated, cultured, characterized and transduced with lentiviral vectors carrying the BDNF exogene. Flow cytometric sorting and G418 resistance selection was conducted to obtain a stable overexpressing-BDNF new cell line BDNF-ADSCs. Different analyses, such as flow cytometry, differentiation capacity, cell proliferation assay, colony forming unit assay, were undertaken to investigate whether BDNF transduction would affect the stemness maintenance of the ADSCs. Meanwhile, western-blot and Real-time PCR were employed to study the anti-inflammation property of the ADSCs and the release of other neurotropic factors induced by BDNF in BDNF-ADSCs.

\section{Materials and Methods}

\section{Isolation of adipose-derived stem cells}

The procedure of isolation fats and cells from C57BL/6 mice has been approved by the Ethics Committee of Dalian University of Technology. ADSCs were isolated from the abdominal fats of $\mathrm{C} 57 \mathrm{BL} / 6$ mice. The abdominal fats were harvested and washed with D-Hank's buffer to remove red blood cells, and then cut into tiny pieces at room temperature (RT) with the fur and any blood vessels dissected away. The pieces were incubated in digestion buffer $(0.1 \%$ collagenase type 1 in Hank's buffer $)$ at $37^{\circ} \mathrm{C}$ with shaking at $120 \mathrm{rpm}$ for $1.5 \mathrm{hrs}$. Then the digested solution was filtered with $70 \mu \mathrm{m}$ cell strainers to remove undigested fragments and ADSCs were collected by centrifugation at $600 \mathrm{~g}$ for $15 \mathrm{~min}$, and then transferred to $75 \mathrm{~cm}^{2}$ culture flasks (Corning) with DMEM/F12 complete medium, containing 10\% fetal bovine serum (FBS), $1 \%$ Penicillin-Stretomycin-Amphotericin B (PSA) and 1\% L-Glutamine. Culture medium was replaced every 2 3 days, and cells were routinely passaged when they reached $80 \%$ confluence. ADSCs passages $4 \sim 6$ were used for different experiments.

\section{Transduction}

BDNF lentiviral vector [pLV[Exp]-EGFP/Neo-EF1A > $\mathrm{mBdnf}$ ] were acquired by restriction endonuclease digestions and gene recombination. 293T cells were co-transfected with a plasmid expressing BDNF, carrying an enhanced green fluorescent protein gene (EGFP) and a neomycin resistance gene (neo) for selective marker to generate BDNF lentiviral particles. The ADSCs, seeded in 6-well plates with a density of $10^{6}$ cells per well, were incubated with $50 \sim 100 * 10^{6}$ lentiviral particles (multiplicity of infection (MOI) of 50 100) for 24 hrs before the supernatant was removed and replaced with fresh growth medium. The ADSCs were observed for EGFP expression under microscope after $48 \mathrm{hrs}$ of transduction. During transduction, polybrene was added $(5 \mu \mathrm{g} / \mathrm{ml})$ to improve the transfection efficiency.

\section{Establishment of stable BDNF-transduced ADSC cell line}

Flow cytometric sorting and G418 resistance selection was conducted for the establishment of stable BDNFtransduced ADSC cell lines. Transduced ADSCs expressing EGFP were sorted by the fluorescence-activated cell sorting (BD, FACSJAZZ) in purity mode, and sorting was gated by forward and side scatter to eliminate the un-transduced cells. The sorted cells were then seeded in 
6-well plates for expansion and G418 was added to inhibit the growth of un-transduced ADSCs. BDNF-ADSCs passages $4 \sim 6$ were also used in the following different experiments.

\section{Flow cytometry}

ADSCs and BDNF-ADSCs were analyzed by flow cytometry (BD FACSCalibur, FACS101) for cell surface marker expression. All the antibodies were purchased from eBioscience Inc. Cells were harvested in $0.25 \%$ trypsin and washed in flow cytometry buffer (PBS, 2\% BSA). The cells with a concentration of $10^{7}$ cells $/ \mathrm{ml}$ were incubated for $90 \mathrm{~min}$ in flow cytometry buffer containing fluorescein conjugated monoclonal antibodies and the cell surface marker antigens respectively: CD29-FITC, CD31FITC, CD34-FITC, Scal-1-FITC, CD11-PE, and CD45-PE. Cells were then fixed with $1 \%$ paraformaldehyde for 30 min and analyzed by BD FACS101. The FSC-H Signal was used to measure cell sizes and cell volume.

\section{Differentiation analysis}

ADSCs and BDNF-ADSCs were cultured in 6-well plates (Costar, New York, USA) at a density of $2 \times 10^{5}$ cells per well. When reached at approximately $90 \%$ confluency, the cells were cultured in osteogenic and adipogenic differentiation medium respectively with medium replaced every 3 days for 3 weeks. Osteogenic differentiation medium was made by complete medium supplemented with $100 \mathrm{nM}$ dexamethasone, $10 \mathrm{mM}$ b-glycerolphosphate, $50 \mu \mathrm{M}$ L-ascorbic acid 2-phosphate sesquimagnesium salt, and $50 \mathrm{ng} / \mathrm{ml}$ L-thyroxine sodium pentahydrate. Adipogenic differentiation medium was made with complete medium supplemented with $5 \mathrm{mg} / \mathrm{ml}$ insulin, $50 \mu \mathrm{M}$ indomethacin, $1 \mu \mathrm{M}$ dexamethasone and $0.5 \mathrm{mM}$ 3-isobutyl-1-methylxanthine. For adipogenic differentiation, the cells were washed with PBS, fixed with 10\% formalin (Sigma) for 20 minutes at RT, washed again with PBS, stained with Oil Red O (Sigma) for 20 minutes at RT, and washed with PBS until wash was clear. For the detection of osteogenesis, the cells were washed with PBS, fixed with $10 \%$ formalin for 20 minutes at RT, washed with deionized (DI) water, stained with Alizarin Red (Sigma) for $20 \mathrm{mi}-$ nutes at RT, and washed with DI water until it was clear. Images were acquired at $10 \times$ for adipogenic differentiation and $4 \times$ for osteogenic differentiation on microscope.

\section{In vitro cell proliferation assay}

ADSCs and BDNF-ADSCs were plated in 24 wells plate at a density of $10^{4}$ cells per well and cultured for 2 weeks. The fold-increase in cell number was analyzed every 2 days throughout the culture period.

\section{Colony forming unit assay}

ADSCs and BDNF-ADSCs were seeded onto $10 \mathrm{~cm}$ cell culture plates (Corning, USA) in 5 replicates at a density of 100 cells per plate. Growth media was replaced every 3 days. After 2 weeks, the cells were washed with PBS, stained with $3 \%$ crystal violet in $100 \%$ methanol for 30 minutes at RT, and then washed with DI water at least 3 times to remove unstained crystal violet. All colonies greater than $2 \mathrm{~mm}$ in diameter were counted.

\section{Extraction of total RNA}

Total RNA was extracted from cultured cells using the RNA extraction kit (Takara, Japan). The concentration and purity of RNA were determined on Nanodrop 2000 (Thermo Scientific, Braunschweig, Germany), and RNA with high purity (the ratios of A260/A230 for the total RNA obtained higher than 2.0, and their ratios of A260/ A280 were more than 1.8) was used for reverse transcription. All RNA samples were stored at $-80^{\circ} \mathrm{C}$ until further use.

\section{Real-time PCR}

RNA was reverse transcribed into cDNA to a final volume of $20 \mu \mathrm{l}$ using PrimeScript ${ }^{\mathrm{TM}}$ RT reagent Kit (Takara, Japan). All cDNA samples were stored at $-80^{\circ} \mathrm{C}$ until further processing. Real-time PCR was performed with the LightCycler (Roche Diagnostics, Mannheim, Germany) to measure and compare the mRNA expression levels of different factors between ADSCs and BDNF-ADSCs. For the real time-PCR analysis, a total volume of $10 \mu 1$ was used following the manual instructions. Briefly, real-time PCR was operated with the two-step method, and melting curves were recorded during the 60 to $95^{\circ} \mathrm{C}$ melting process with continual fluorescence data acquisition. The melting curves were generated during denaturation step at $95^{\circ} \mathrm{C}$ followed by the cooling of PCR products at $60^{\circ} \mathrm{C}$ and the fluorescence signals were collected continuously from 60 to $95^{\circ} \mathrm{C}$ as the temperature increased at $0.2^{\circ} \mathrm{C}$ per second. All reactions were repeated at least three times. Primer sequences used are listed in Table 1 . The $2^{-\triangle \triangle C T}$ Method was used for the quantity calculations.

\section{Western-blot}

For western blot analysis, cell pellets were suspended in radioimmunoprecipitation assay (RIPA) buffer (P0013B; Beyotime) containing PMSF (ST506; Beyotime), and samples were homogenized in a glass homogenizer at $4^{\circ} \mathrm{C}$ for $20 \mathrm{~min}$. Insoluble materials were pelleted by centrifu- 
Table 1. Sequences of mouse specific primers used in real-time PCR analysis

\begin{tabular}{|c|c|c|}
\hline Gene & Forward primer $\left(5^{\prime}-3^{\prime}\right)$ & Reverse primer $\left(5^{\prime}-3^{\prime}\right)$ \\
\hline BDNF & 5'-GAAGGCTGCAGGGGCATAGACAAA-3' & 5'-TACACAGGAAGTGTCTATCCTTATG-3' \\
\hline TNF- $\alpha$ & 5'-CСАСАТСТСССТССАGААAА-3' & 5'-AGGGTCTGGGCCATAGAACT-3' \\
\hline IL-1a & 5'-СТTССТСАСССТGАGTCACTTGC-3' & 5'-GTGGCTCCACTAGGGTTTGCTCT-3' \\
\hline IL-1b & 5'-CCCAACTGGTACATCAGCACCTC-3' & 5'-GACACGGATTCCATGGTGAAGTC-3' \\
\hline IL-6 & 5'-CGGAGAGGAGACTTCACAGAGGA-3' & 5'-TTTCCACGATTTCCCAGAGAACA-3' \\
\hline IL10 & 5'-GCCCTTTGCTATGGTGTCCTTTC-3' & 5'-TCССТGGTTTCTCTTCCCAAGAC-3' \\
\hline IKK & 5'-CCAGACTCCAAGGTGGTGTT-3' & 5'-TGCAGATCACAGGCAGAAAC-3' \\
\hline$\beta$-actin & GCCAACCGTGAAAAGAT & AGAGCATAGCCCTCGTAGAT \\
\hline
\end{tabular}
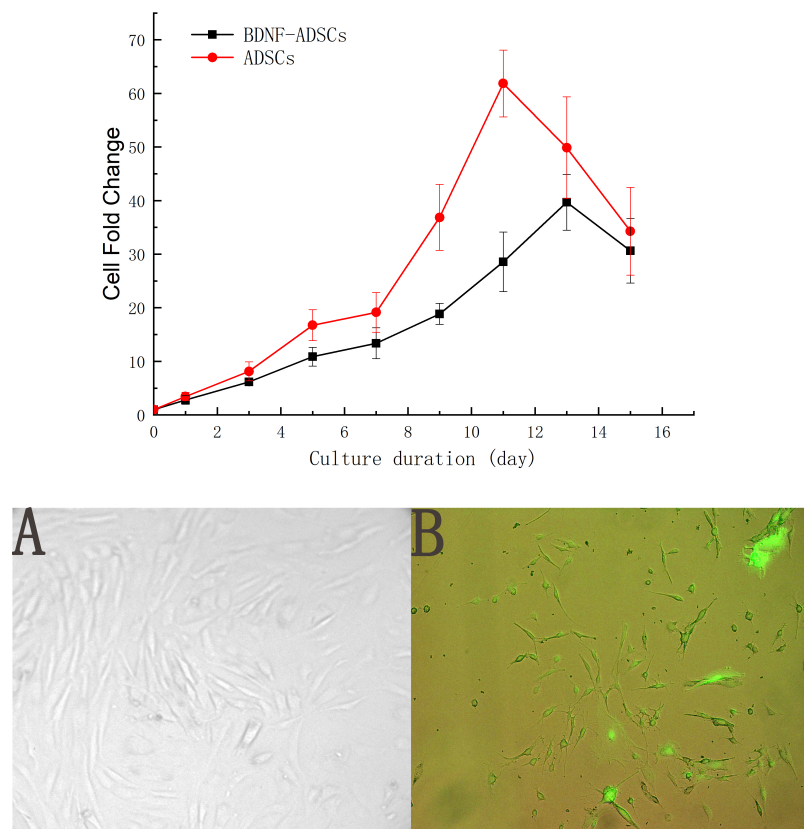

Fig. 1. Cell morphology and growth profiles of ADSCs and BDNFADSCs.

gation for $15 \mathrm{~min}$ at $12,000 \mathrm{rpm}$. The amounts of proteins from ADSCs or BDNF-ADSCs cells were normalized by BCA method (P0012; Beyotime). The samples were first loaded onto PAGE 15\% Acr-Bis gel and then transferred to polyvinylidene fluoride membranes (PVDF; CA, USA). Membranes loaded with samples were incubated with antibodies and washed, and images were captured using FluorChem HD2 (Alpha Innotech Corp, San Leandro, CA, USA) according to manufacturer's instructions. Primary antibodies used were rabbit anti-p75 NGF receptor polyclonal antibody (bs-7122R; Bioss), rabbit anti-CNTF antibody (bs-1272R; Bioss), rabbit anti-BDNF antibody (ab108319; Abcam), rabbit anti-bFGF antibody (bs-0217R; Bioss).

\section{Statistical analysis}

Data are presented as mean \pm SD from at least 3 independent cell samples. Student t-test and one-way analysis of variance (ANOVA) were used to analyze the data. $\mathrm{p}<0.05$ was considered as statistically significant.

\section{Results}

\section{Cell morphology and growth kinetics of ADSCs and BDNF-ADSCs}

As shown in Fig. 1, the cell morphology, which is fibroblasts-like, of the BDNF-ADSCs was consistent with the un-transfected ADSCs, moreover, the green fluorescence shown by BDNF-ADSCs proved that ADSCs was successfully transfected with BDNF plasmid. Meanwhile, measurement of the proliferative capacity of the BDNF-ADSCs demonstrated that the growth curve of the BDNF-ADSCs is like that of ADSCs in general although the maximum fold change is not as high as that of ADSCs.

\section{Surface antigen analysis on ADSCs and BDNF-ADSCs by flow cytometry}

Both ADSCs and BDNF-ADSCs were cultured, stained and analyzed for cell surface marker expression. ADSC surface markers CD29, Scal; hematopoietic markers CD34 and CD45; phagocytic lineage marker CD1lb; and endothelial marker CD31 were tested by flow cytometry. As shown in Fig. 2A, both ADSCs and BDNF-ADSCs were positive for specific antigen markers of ADSCs like CD29 and NK/ILC markers like ScaI-1, while they were negative for endothelial marker CD31, hematopoietic markers CD45, CD34 and macrophage marker CD11b. Meanwhile, the cell sizes of both ADSCs and BDNFADSCs were also measured based on the forward scatter signal of flow cytometry, and data in Fig. 2B demonstrated that there were no significant differences between the cell sizes of ADSCs and BDNF-ADSCs ( $\mathrm{p}=0.13$, t-test, $\mathrm{n}=6)$. 

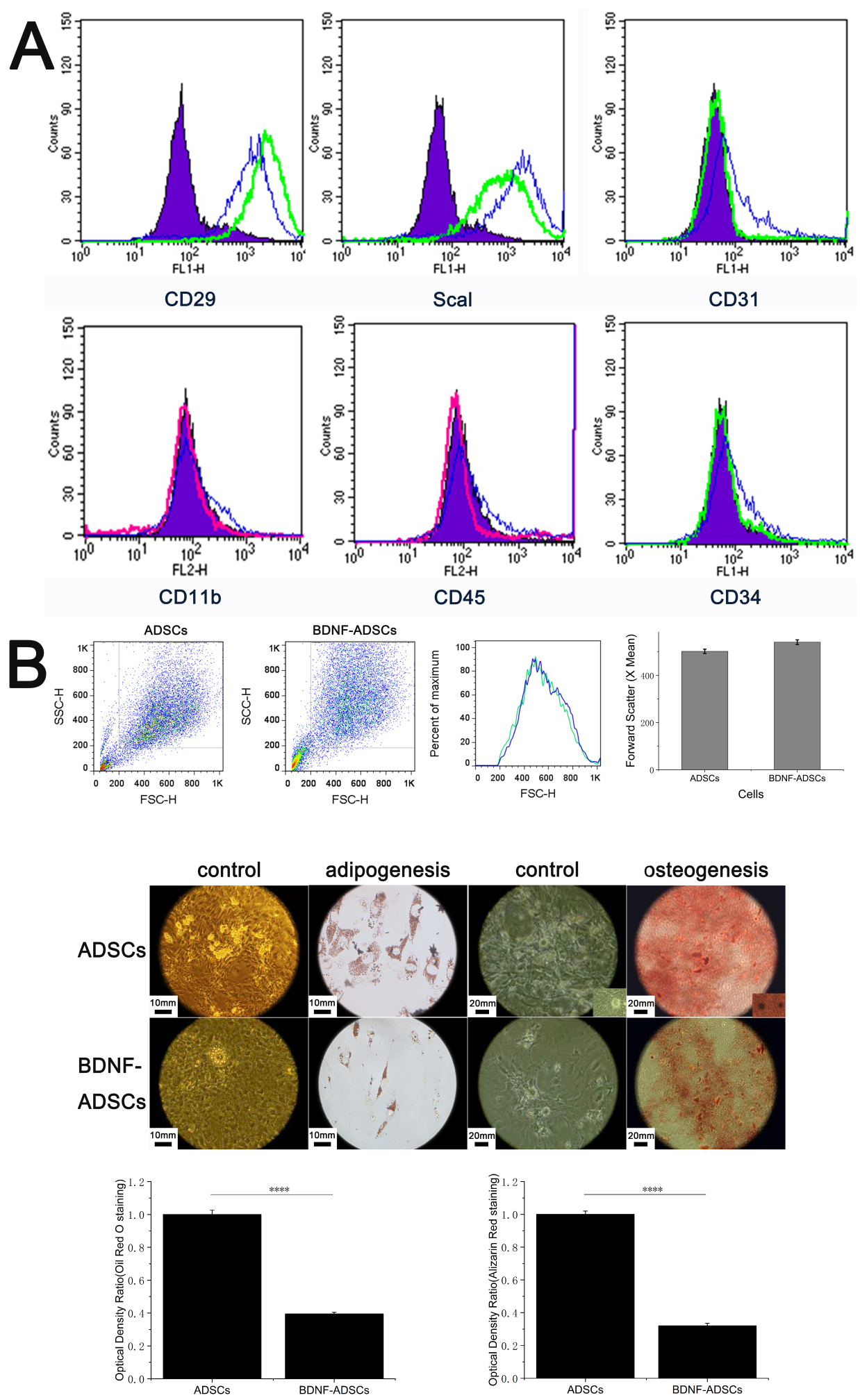

Fig. 2. (A) Cell surface marker profiles of ADSCs and BDNF-ADSCs. Murine ADSCs and BDNF-ADSCs were stained with stem cells' surface markers CD29, Sca1; hematopoietic markers CD34 and CD45; phagocytic lineage marker CD11b; and endothelial marker CD31 and analyzed by flow cytometry. Green lines represent $A D S C s$ stained with CD29-FITC, CD31-FITC, CD34-FITC, Scal-1-FITC; Red lines show ADSCs stained with CD45-PE, CD11b-PE; Blue lines represent BDNF-ADSCs stained with the same antibody as ADSCs for compariso, and purple area shows the respective IgG isotype control for each antibody. (B) Cell size based on forward scatter signal of flow cytometry $(p=0.13$, t-test, $\mathrm{n}=6$ ).
Fig. 3. Qualitative and quantitative adipogenic and osteogenic differentiation of ADSCs and BDNF-ADSCs. Cells were incubated in adipogenic or osteogenic differentiation media for 21 days and stained with Oil Red $\mathrm{O}$ and Alizarin Red respectively. The inserts in osteogenensis of ADSCs panels emphasize the calcium nodus produced by the ADSCs. $* * * *$ means much statistically significant $(\mathrm{p}<0.0001, \mathrm{n}=3)$.

\section{Differentiation assays for ADSCs and BDNF-ADSCs}

BDNF-ADSCs and ADSCs were cultured in adipogenic and osteogenic differentiation medium respectively for 3 weeks, and then stained with Oil Red O and Alizarin Red to test their differentiation efficiency. The results (Fig. 3) demonstrated that BDNF-ADSCs preserve the ability of multi-lineage potential, although the potential is not as strong as the un-transduced ADSCs. 


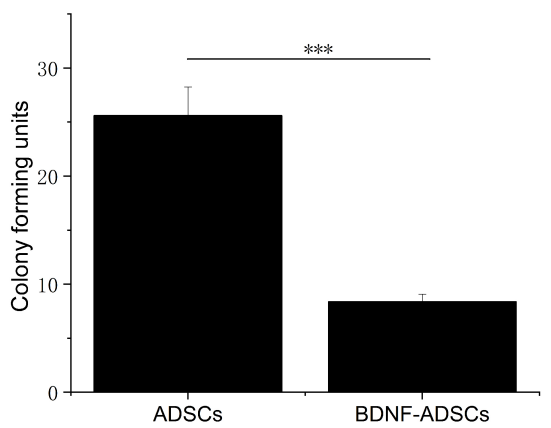

Fig. 4. Colony forming unit assays for ADSCs and BDNF-ADSCs. A total of 100 cells were plated on $10 \mathrm{~cm}$ cell culture plates and incubated for 14 days. Cells were stained with 3\% crystal violet, and colonies $2 \mathrm{~mm}$ or larger in diameter were counted. ${ }^{* * *}$ means much statistically significant $(\mathrm{p}<0.0001, \mathrm{n}=3)$.

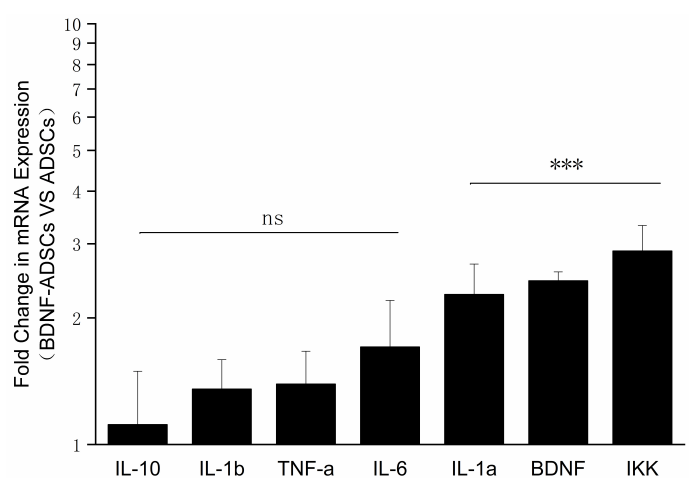

Fig. 5. Real-time PCR analysis of cytokine and chemokine profiles of BDNF-ADSCs vs ADSCs. ns means the expression levels are not statistically significant compared to those of ADSCs ( $p>0.05, n=3)$. ***means the expression levels are highly statistical significant compared to those of ADSCs $(p<0.001, n=3$ ).

\section{Colony forming unit assay for ADSCs and BDNF-ADSCs}

ADSCs and BDNF-ADSCs were assessed for their self-renewal ability by the colony forming unit (CFU) assay. All colonies greater than $2 \mathrm{~mm}$ in diameter were counted. Fig. 4 showed that BDNF-ADSCs still has the self-renewal capacity, although the number and density of the units formed are smaller in comparison to the un-transfected ADSCs control.

\section{Real-time polymerase chain reaction}

Real-time polymerase chain reaction (PCR) assay was performed to analyze the levels of BDNF, related signaling pathways gene IKK and inflammatory cytokines (IL-1a，IL-1b，TNF-a，IL-6 and IL-10) expressed by ADSCs and BDNF-ADSCs. The housekeeping gene b-actin was used as internal reference. Quantification was calculated using $2^{-\triangle M C T}$ method. As illustrated in Fig. 5, the

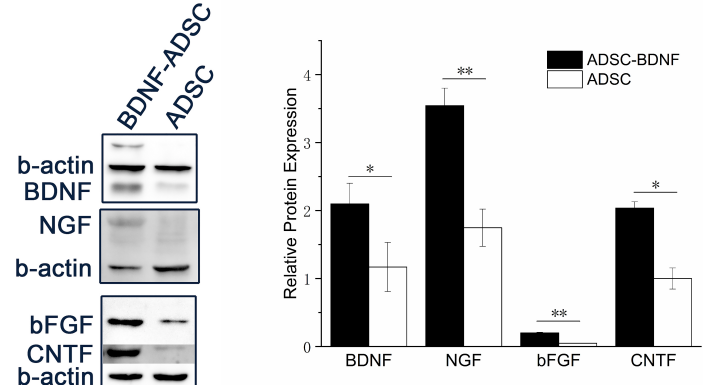

Fig. 6. Western-Blot analyses for murine protein expression of NGF, BDNF, bFGF and CNTF in BDNF-ADSCs and ADSCs. *means statistically significant $(p<0.05, n=3)$, and ${ }^{* *}$ means much statistically significant $(p<0.01, n=3)$.

mRNA expression levels of BDNF, related signaling pathways gene IKK and anti-inflammatory cytokine IL-1a are significantly higher in BDNF-ADSCs in comparison to those expressed in un-transfected ADSCs control.

\section{Western-blot analysis}

The protein expressions of neurotrophic factors BDNF, NGF, CNTF, bFGF in BDNF-ADSCs and ADSCs were normalized to control $\beta$-actin. The grayscale of bands were semi-quantified by AlphaView Software, Version 3.4.0.0. Western blot analysis data as shown in Fig. 6 revealed that all the neurotrophic factors BDNF, NGF, CNTF, bFGF tested had significant increased expression in BDNF-ADSCs while compared to those in ADSCs.

\section{Discussion}

The present study was carried out to investigate whether the ADSCs transduced with BDNF still keep their stemness abilities and characteristics, and whether ADSCs, alongside acting as the gene delivery vehicle of BDNF, could contribute synergistic therapeutic effects to those of BDNF. The results demonstrated that the BDNF-ADSCs displayed all the characteristics that defined for mesenchymal stem cells in vitro $(8,14)$, which are mononuclear cell population adherent to plastic culture surfaces, fibroblast-like and spindle shaped, possess osteogenic and adipogenic differentiation potential (Fig. 3). Meanwhile, the BDNF-ADSCs, like MSCs, are positive for stem cell surface markers CD29 and ScaI, and negative for hematopoietic markers CD45, CD31, CD34 and macrophage marker CD11b (Fig. 2) (15, 16). However, The data from Fig. 1, 3 and 4 showed that, while BDNF-ADSCs still proliferate, differentiate and could form single colonies like un-transduced ADSCs, the rate was slowed down which 
could be explained by the increased burden of the addition of exogenous BDNF $(17,18)$, while it has been demonstrated that lentiviral eGFP transduction did not affect the proliferation, differentiation and CFU forming abilities of ADSCs (19).

Real-time PCR analysis (Fig. 5) revealed that, along with the increased BDNF mRNA expression level in BDNF-ADSCs, the mRNA expression level of interleukin (IL)-1 $\alpha$ and inhibitor of nuclear factor kappa-B kinase (IKK) were also significantly increased $(\mathrm{p}<0.001, \mathrm{n}=3)$ in comparison with those expressed in un-transduced ADSCs. IL- $1 \alpha$ is one of the most well studied member of interleukin family, and is recognized as an important player in cancer and other inflammatory diseases $(20,21)$. IL-1 $\alpha$ is thought to be a dual-function cytokine that represents both nuclear and extracellular functions and active in inflammation and immune response processes (22). Research conducted by Redondo-Castro et al. (23) found out that mesenchymal stem cells pre-conditioned with IL-1 $\alpha$ increases expression of trophic factor G-CSF and induces a reduction in the secretion of inflammatory mediators in LPS-activated microglial cells, which suggests that BDNF-ADSCs with a higher expression of IL-1 $\alpha$ may be beneficial to damaged neuronal recovery and survival. IKK plays an important role in the signaling pathway induced by the combination of BDNF and tyrosine kinase receptor $\mathrm{B}(\operatorname{TrkB})$. Phosphorylation of IKK activates nucleus survival factor $\mathrm{NFkB}$, thus exerting its anti-apoptotic effect $(24,25)$.

The results obtained from western-blot experiment (Fig. 6) showed that the expression of other neurotrophic factors, such as NGF, CNTF, bFGF, was also increase in BDNF-ADSC when compared to that of wild type ADSCs. Besides the anti-apoptotic effects, BDNF has been known to help induce the release of other neurotrophic factor (26), the combination of these neurotrophic factors will have an more lasting and extensive protection effects of neuronal cells.

In conclusion, the transduction of BDNF into ADSCs did not change the stem cell characteristics of ADSCs, Moreover, the BDNF helped induce expression of the immune response factors like IKK and IL- $1 \alpha$, and other neurotrophic factors like NGF, CNTF, bFGF. ADSCs could break through the blood-brain barrier and migrate to the damaged tissue, and its low immunogenicity could reduce immune rejection. Therefore, the ADSCs are ideal gene delivery vectors for BDNF. Meanwhile, the anti-inflammation and immune regulation properties of ADSCs could be synergistic to the therapeutic effects of BDNF.

\section{Acknowledgments}

This work was supported by the National Natural Science Foundation of China (grant number: 31400840).

The authors thank School of Life Science and Medicine for help with performing the real time PCR and western blot assays in this study.

\section{Potential Conflict of Interest}

The authors have no conflicting financial interest.

\section{References}

1. Chen R, Yin XB, Peng CX, Li GL. Effect of brain-derived neurotrophic factor on c-jun expression in the $\mathrm{rd}$ mouse retina. Int J Ophthalmol 2012;5:266-271

2. Ernfors P, Wetmore C, Olson L, Persson H. Identification of cells in rat brain and peripheral tissues expressing mRNA for members of the nerve growth factor family. Neuron 1990;5:511-526

3. Nibuya M, Morinobu S, Duman RS. Regulation of BDNF and trkB mRNA in rat brain by chronic electroconvulsive seizure and antidepressant drug treatments. J Neurosci 1995; 15:7539-7547

4. Amendola T, Fiore M, Aloe L. Postnatal changes in nerve growth factor and brain derived neurotrophic factor levels in the retina, visual cortex, and geniculate nucleus in rats with retinitis pigmentosa. Neurosci Lett 2003;345:37-40

5. Mannermaa E, Vellonen KS, Urtti A. Drug transport in corneal epithelium and blood-retina barrier: emerging role of transporters in ocular pharmacokinetics. Adv Drug Deliv Rev 2006;58:1136-1163

6. Tao W, Wen R, Goddard MB, Sherman SD, O'Rourke PJ, Stabila PF, Bell WJ, Dean BJ, Kauper KA, Budz VA, Tsiaras WG, Acland GM, Pearce-Kelling S, Laties AM, Aguirre GD. Encapsulated cell-based delivery of CNTF reduces photoreceptor degeneration in animal models of retinitis pigmentosa. Invest Ophthalmol Vis Sci 2002;43:32923298

7. Tian C, Weng CC, Yin ZQ. BDNF improves the efficacy ERG amplitude maintenance by transplantation of retinal stem cells in RCS rats. Adv Exp Med Biol 2010;664:375-384

8. Dominici M, Le Blanc K, Mueller I, Slaper-Cortenbach I, Marini F, Krause D, Deans R, Keating A, Prockop Dj, Horwitz E. Minimal criteria for defining multipotent mesenchymal stromal cells. The International Society for Cellular Therapy position statement. Cytotherapy 2006;8: 315-317

9. Semon JA, Zhang X, Pandey AC, Alandete SM, Maness C, Zhang S, Scruggs BA, Strong AL, Sharkey SA, Beuttler MM, Gimble JM, Bunnell BA. Administration of murine stromal vascular fraction ameliorates chronic experimental autoimmune encephalomyelitis. Stem Cells Transl Med 2013;2:789-796

10. Jin HJ, Bae YK, Kim M, Kwon SJ, Jeon HB, Choi SJ, Kim SW, Yang YS, Oh W, Chang JW. Comparative analysis of 
human mesenchymal stem cells from bone marrow, adipose tissue, and umbilical cord blood as sources of cell therapy. Int J Mol Sci 2013;14:17986-18001

11. Jankowiak W, Kruszewski K, Flachsbarth K, Skevas C, Richard G, Rüther K, Braulke T, Bartsch U. Sustained neural stem cell-based intraocular delivery of CNTF attenuates photoreceptor loss in the nclf mouse model of neuronal ceroid lipofuscinosis. PLoS One 2015;10:e127204

12. Kishimoto $S$, Inoue $\mathrm{K}$, Nakamura $\mathrm{S}$, Hattori $\mathrm{H}$, Ishihara $M$, Sakuma $M$, Toyoda S, Iwaguro $H$, Taguchi I, Inoue T, Yoshida K. Low-molecular weight heparin protamine complex augmented the potential of adipose-derived stromal cells to ameliorate limb ischemia. Atherosclerosis 2016;249: 132-139

13. Utsunomiya $T$, Shimada $M$, Imura $S$, Morine $Y$, Ikemoto T, Mori H, Hanaoka J, Iwahashi S, Saito Y, Iwaguro H. Human adipose-derived stem cells: potential clinical applications in surgery. Surg Today 2011;41:18-23

14. Mehrabani D, Mehrabani G, Zare S, Manafi A. Adipose-derived stem cells (ADSC) and aesthetic surgery: a mini review. World J Plast Surg 2013;2:65-70

15. Braun J, Kurtz A, Barutcu N, Bodo J, Thiel A, Dong J. Concerted regulation of CD34 and CD105 accompanies mesenchymal stromal cell derivation from human adventitial stromal cell. Stem Cells Dev 2013;22:815-827

16. Zhang X, Bowles AC, Semon JA, Scruggs BA, Zhang S, Strong AL, Gimble JM, Bunnell BA. Transplantation of autologous adipose stem cells lacks therapeutic efficacy in the experimental autoimmune encephalomyelitis model. PLoS One 2014;9:e85007

17. Connor RI, Mohri H, Cao Y, Ho DD. Increased viral burden and cytopathicity correlate temporally with CD4+ Tlymphocyte decline and clinical progression in human im- munodeficiency virus type 1-infected individuals. J Virol 1993;67:1772-1777

18. Mastrangeli A, Danel C, Rosenfeld MA, StratfordPerricaudet L, Perricaudet M, Pavirani A, Lecocq JP, Crystal RG. Diversity of airway epithelial cell targets for in vivo recombinant adenovirus-mediated gene transfer. J Clin Invest 1993;91:225-234

19. Madonna R, Bolli R, Rokosh G, De Caterina R. Long-term engraftment and angiogenic properties of lentivirally transduced adipose tissue-derived stromal cells. Mol Biotechnol 2013;54:13-24

20. Rider P, Carmi Y, Voronov E, Apte RN. Interleukin- $\alpha$. Semin Immunol 2013;25:430-438

21. Dinarello CA. Interleukin-1 in the pathogenesis and treatment of inflammatory diseases. Blood 2011;117:3720-3732

22. Bertheloot D, Latz E. HMGB1, IL-1 $\alpha$, IL-33 and S100 proteins: dual-function alarmins. Cell Mol Immunol 2017;14: 43-64

23. Redondo-Castro E, Cunningham C, Miller J, Martuscelli L, Aoulad-Ali S, Rothwell NJ, Kielty CM, Allan SM, Pinteaux E. Interleukin-1 primes human mesenchymal stem cells towards an anti-inflammatory and pro-trophic phenotype in vitro. Stem Cell Res Ther 2017;8:79

24. Barbacid M. The Trk family of neurotrophin receptors. J Neurobiol 1994;25:1386-1403

25. Hetman M, Kanning K, Cavanaugh JE, Xia Z. Neuroprotection by brain-derived neurotrophic factor is mediated by extracellular signal-regulated kinase and phosphatidylinositol 3-kinase. J Biol Chem 1999;274:22569-22580

26. Wilson RB, Kunchithapautham K, Rohrer B. Paradoxical role of BDNF: $\mathrm{BDNF}+/-$ retinas are protected against light damage-mediated stress. Invest Ophthalmol Vis Sci 2007; 48:2877-2886 